\title{
Comparison of the Automated Pattern-Noise (PANO) Glaucoma Test with the HFA, an FDT Stimulus, and the Fundus Area Cup-to-disk Ratio
}

\author{
Thomas Hannen ${ }^{1}$, Sylvain El-Khoury ${ }^{2}$, Rajesh Patel ${ }^{3}$, Faustin Ngounou ${ }^{4}$, Paul-Rolf Preußner ${ }^{5}$
}

\begin{abstract}
Aim and objective: To compare the results of a new automated glaucoma test—Pattern-Noise (PANO)—-to the Humphrey Visual Field Analyzer-II (HFA), the fundus area cup-to-disk ratio (CDR), and a frequency doubling technology (FDT) stimulus.

Materials and methods: This was a prospective study performed in the West-Region of Cameroon. Two hundred and nineteen eyes of 122 adult patients were included with a clinical suspicion of normal-tension or primary open-angle glaucoma and no other major ocular pathology. Eyes were examined with PANO, HFA (24-2 SITA standard), and FDT-stimulus in a randomized order followed by clinical assessment of the CDR. Results: Parametric correlation of the mean contrast threshold of PANO with the mean contrast threshold of FDT-stimulus, total deviation of HFA, and area CDR was $0.94,-0.85$, and 0.62 , respectively $(p<0.001$ for all values). Spatial distribution of sensitivity thresholds is highly correlated $(p<0.001)$ at all points in the visual field between PANO and HFA. With cut-off values of $3 \pm 1 \mathrm{~dB}$ for HFA mean deviation and $4 \pm 1$ for PANO mean contrast threshold and after eliminating borderline cases, PANO's sensitivity was $95 \%$ and specificity $60 \%$. The mean patient age was $45.2 \pm 15.8$ years. Mean thresholds of PANO and FDT-stimulus decreased with increasing age. Mean examination time was $7.1 \pm 1.8$ minutes for PANO, $5.9 \pm 1.3$ minutes for HFA, and $4.7 \pm 1.3$ minutes for FDT-stimulus. The mean percentage of false-positives per examination was $4.95 \%$ for PANO, $4.62 \%$ ( $p=0.025)$ for FDT-stimulus, and $2.10 \%$ for HFA.

Conclusion: The results showed that PANO was successful in suspecting the presence of glaucoma. Pattern-Noise examination led to findings that were significantly correlated to HFA, FDT stimulus, and area CDR. Some patterns of defect were also correlated. Furthermore, PANO showed a reasonable examination time and error rate.

Clinical significance: Affordable and robust visual field devices are lacking in large parts of the developing world. Comparing them to established methods is a prerequisite to their clinical use.

Keywords: Frequency doubling technology, Glaucoma, Healthcare research, Humphrey field analyzer, Pattern-Noise, Visual field.

Journal of Current Glaucoma Practice (2021): 10.5005/jp-journals-10078-1317
\end{abstract}

\section{INTRODUCTION}

Glaucoma is the primary cause of irreversible blindness in the world. ${ }^{1}$ A lack of healthcare infrastructure in sub-Saharan Africa coincides with a higher prevalence of glaucoma in the black population. Information about the prevalence of glaucoma in sub-Sahara Africa is sparse, but the notion of increased glaucoma prevalence compared with the Caucasian race is generally agreed on. ${ }^{2-6}$ One study performed in Western Cameroon revealed 10 times higher glaucoma prevalence when compared with the German population. ${ }^{4}$

Diagnosis of glaucoma takes into account several components including the measurement of intraocular pressure, the optic nerve head morphology, and perimetry. The latter represents a functional assessment that is especially relevant in the detection of disease progression in eyes known to have a visual field defect at baseline. ${ }^{7}$ The combination of these components is essential for the proper management of the disease. Commercially available perimetries, however, are expensive and difficult to maintain. As such, several initiatives were taken to develop visual field examinations that are freely available online and that can be used as a screening method (Damato Multifixation Campimeter Online, ${ }^{8}$ Peristat, ${ }^{9}$ Visual Fields Easy $\left.{ }^{10}\right)$. However, these developments were not aimed to have a

\footnotetext{
1,5 Department of Ophthalmology, University Medical Center Mainz, Langenbeckstraße 1, Mainz, Germany

${ }^{2}$ Department of Ophthalmology, University Medical Center Mainz, Langenbeckstraße 1, Mainz, Germany; Department of Pediatrics and Retina, Fondation Ophtalmologique Adolphe de Rothschild, Paris, France

${ }^{3,4}$ Department of Ophthalmology, Presbyterian Eye Hospital, Bafoussam, Cameroon

Corresponding Author: Sylvain El-Khoury, Department of Ophthalmology, University Medical Center Mainz, Langenbeckstraße 1, Mainz, Germany; Department of Pediatrics and Retina, Fondation Ophtalmologique Adolphe de Rothschild, Paris, France, Phone: +33(0)148036568, e-mail: sylvain_elkhoury@yahoo.com

How to cite this article: Hannen T, El-Khoury S, Patel R, et al. Comparison of the Automated Pattern-Noise (PANO) Glaucoma Test with the HFA, an FDT Stimulus, and the Fundus Area Cup-to-disk Ratio. J Curr Glaucoma Pract 2021;15(3):132-138.

Source of support: This study was funded by the Research Grant for Tropical Ophthalmology of the Deutsche Ophthalmologische Gesellschaft (DOG-German Ophthalmological Society)

Conflict of interest: Author TH received the Research Grant for Tropical Ophthalmology of the Deutsche Ophthalmologische Gesellschaft (DOG). All other authors declare that they have no conflict of interest
}

(O) The Author(s). 2021 Open Access This article is distributed under the terms of the Creative Commons Attribution 4.0International License (https://creativecommons. org/licenses/by-nc/4.0/), which permits unrestricted use, distribution, and non-commercial reproduction in any medium, provided you give appropriate credit to the original author(s) and the source, provide a link to the Creative Commons license, and indicate if changes were made. The Creative Commons Public Domain Dedication waiver (http://creativecommons.org/publicdomain/zero/1.0/) applies to the data made available in this article, unless otherwise stated. 
high sensitivity ${ }^{8-10}$ and therefore cannot be used for early diagnosis or for a reliable progression detection.

Our research group has presented in a previous publication a new device for the diagnosis of glaucoma called Pattern-Noise (PANO). ${ }^{11}$ The device was primarily developed for use in low-income countries. As such, PANO can be constructed on the spot with parts easily interchangeable and the software is freely available for low-income countries. A primary small study on PANO showed a significant correlation between the detected contrast threshold and the cup-to-disk ratio, a feasible examination time, and a good patient comprehension. ${ }^{11}$

The present paper aims to compare the results of the PANO examinations in glaucoma suspect eyes with the Humphrey Visual Field Analyzer (HFA II, Carl Zeiss Meditec, Jena, Germany), the FDT stimulus on a PANO device, and the fundus area cup-to-disk ratio (CDR) of the optic nerve.

\section{Materials and Methods}

This is a prospective study that was approved by the institutional review board (IRB) of the Presbyterian Church in Cameroon (PCC) Eye Services and was in accordance with the Helsinki Declaration of 1975.

The study was performed at the main hospital of the PCC Eye Services located in Douala, Cameroon. This center is one of the largest eye care providers in the country. Between August 13, 2013, and September 19, 2013, all patients above 18 years of age presenting with a clinical suspicion of normal-tension (NTG) or primary open-angle glaucoma (POAG) were invited to participate in the study. All patients were of black ethnicity. Clinical suspicion was raised when fundoscopy showed a characteristic glaucomatous optic disk (local notching or thinning of the neuroretinal rim, ISNT rule not respected, large cup-to-disk ratio, peripapillary hemorrhage, and asymmetry between optic disks). Many patients were already on topical glaucoma treatment despite having no prior standard automated perimetry (SAP) examination, due to its unavailability in the facility. Written consent was obtained from all patients before inclusion, after having read an explanatory text of the study, available to them in English and French. Subject exclusion criteria were any other major ocular pathology, such as cataract, corneal or retinal disease, or any other pathology limiting the ability to reliably perform a visual field examination.

The study sequence was as follows: (1) Refraction, correcteddistance visual acuity (CDVA), slit-lamp biomicroscopy of the anterior segment, and intraocular pressure (IOP). (2) PANO, HFA, and FDT visual field examination in randomized order. The laterality of the examined eye was included in the randomization. All visual field examinations were performed in the same darkened, airconditioned room and a pause of a minimum of 3 minutes between examinations was allowed as a recreation. (3) Dilated fundoscopy with a notation of the area cup-to-disk ratio. (4) A handing out of the result sheet to the patients and discussion of the results with the patients and their respective physicians. Steps 2, 3, and 4 were performed by the same examiner (TH).

Before the PANO test was performed the laptop was given a 15-minute warm-up time and patients were corrected for far vision. Patients were then briefed on how to perform the examination and were allowed a practice time of at least 2 minutes.

Statistical analysis was performed with a separate program written by one of the authors (PRP). Descriptive statistics were reported as mean \pm standard deviation for continuous variables and as a percentage for categorical variables. $p$ value $<0.05$ was considered to be statistically significant.

Due to missing normative data for a sub-Saharan population, the preliminary mean deviation cut-off value for discriminating glaucomatous field damage was based on literature and was for HFA $-3 \pm 1 \mathrm{~dB}^{12}$ and for PANO $4 \pm 1$. $^{11}$ Thus for HFA normal, borderline glaucoma and glaucoma were considered $>-2$, between -2 and -4 , and $<-4$ mean total deviation, respectively, and for PANO $<3$, between 3 and 5, $>5$ mean threshold, respectively.

\section{PANO}

For a detailed description of the hardware, software and the result sheet of the PANO device refer to El-Khoury et al. ${ }^{11}$ The main principles of PANO are described in brief: PANO is a visual field device that measures the sensitivity threshold in detecting a target of a flickering contrast, i.e., it measures the contrast sensitivity. Targets are squares and have a size of approximately $5^{\circ} \times 5^{\circ}$ (degrees of $\operatorname{arc}$ ) and are made of bright and dark pixels that flicker with a frequency of $18 \mathrm{~Hz}$. The density of the pixels is adjusted to the hill of vision and decreases from the center $(72 \times 72)$ to the periphery $(9 \times 9)$. In essence, PANO tests the level of contrast (Michelson contrast) between pixels (contrast range: 2-64, with 2 being the lowest contrast and 64 being the highest). The level of the contrast is derived from the 128-luminance degrees of the screen. The average luminance of the targets equals to the luminance of the background. Targets are displayed on 54 possible locations that can be directly compared with the 24-2 analyzer protocol of the HFA-II.

The testing strategy is as follows: The patient fixates the center of the screen, which displays a green fixation target, and is asked to respond by clicking on a mouse button when perceiving a target. Targets are displayed for 0.5 seconds at different positions on the screen and the patient has between 0.1 and 0.5 seconds after the appearance of the target to respond. Positive feedback (brightening of the central fixation point) in case of a correct response, and negative feedback (emission of a high pitch sound) are incorporated into the software to increase patient focus. An error is considered if the patient executes a motor response outside this time frame. The percentage of errors per number of motor responses during one examination is displayed on the result sheet and represents the percentage of false-positives.

The PANO software was written by one of the authors (PRP). The hardware consists of a commercial laptop (Aspire E1-571 Acer Inc.), a mechanical frame to hold the laptop, a modified PC mouse, a modified chin rest with a $4 \mathrm{D}$ rotatable magnifying glass, and a laser printer. Refer to Figure 1 for an exemplary result sheet of a PANO examination.

\section{HFA}

The HFA-II 740 (Carl Zeiss Meditec, Jena, Germany) was used with a SITA (Swedish interactive threshold algorithm) Standard strategy and a 24-2 analyzer protocol. The HFA is a white-on-white SAP. It is considered to be the clinical standard. ${ }^{13}$

\section{FDT-stimulus}

The PANO device was used to present a target stimulus that is very similar to the stimulus of the Humphrey FDT (Carl Zeiss Meditec, Jena, Germany). Targets were vertical sine-wave grating alternating with a frequency of $18 \mathrm{~Hz}$ between dark and bright. ${ }^{14}$ The tested value is the level of contrast, ranging from 1 to 16 with increasing contrast. Testing strategy and target arrangement were identical to PANO. 


\section{Area Cup-to-disk Ratio}

This is the ratio of the area of the cup divided by the area of the disk. It has the advantage of being less prone to variations of the optic disk size than the linear cup-to-disk ratio. It was calculated by using estimates of the superior $(s)$, temporal $(t)$, inferior $(i)$, and nasal margin widths $(n)$ as a fraction of the total disk diameter with the following formula: ${ }^{4}$

$$
\text { Area } \mathrm{CDR}=\pi *(1-n-t) *(1-i-s)
$$

\section{Results}

Overall, 219 eyes of 122 patients were included in the analysis. $57.4 \%$ were males and $42.6 \%$ were females. Mean patient age was $45.2 \pm$ 15.8 years (range: $18-71$ years), $36 \%$ of patients were older than 55 years. Mean IOP was $19.2 \pm 5.7 \mathrm{~mm} \mathrm{Hg}$ (median: 18; range 9-44).

\section{OD 302636}

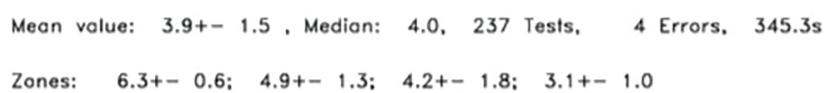

$$
3.1
$$$$
3.0 \quad 2.0
$$$$
2.0 \quad 4.0 \quad 3.4
$$$$
\begin{array}{lll}
2.0 & 2.0 & 3.0
\end{array}
$$$$
\begin{array}{llllllll}
3.0 & 2.0 & 2.0 & 4.0 & 5.0 & 3.0 & 2.0 & 3.0
\end{array}
$$$$
\begin{array}{lll}
4.0 & 2.0 & 2.0
\end{array}
$$$$
\begin{array}{lllll}
4.0 & 3.0 & 3.0 & 3.0 & 7.0
\end{array}
$$$$
\begin{array}{lllll}
4.0 & 3.0 & 4.0 & 4.0 & 6.0
\end{array}
$$$$
\begin{array}{llll}
3.0 & 2.0 & 3.0 & 7.0
\end{array}
$$$$
\begin{array}{lll}
4.0 & 5.0 & 6.0
\end{array}
$$$$
5.0 \quad 5.0
$$$$
\begin{array}{llll}
5.0 & 3.0 & 2.0 & 3.0
\end{array}
$$$$
\begin{array}{llll}
6.0 & 5.0 & 4.0 & 2.0
\end{array}
$$$$
\begin{array}{llll}
6.0 & 5.0 & 8.0 & 4.0
\end{array}
$$$$
\begin{array}{llll}
6.0 & 6.0 & 4.0 & 3.0
\end{array}
$$$$
\begin{array}{lll}
4.0 & 3.0 & 3.0
\end{array}
$$

4.4

$4.0 \quad 4.0$

\section{6}

Fig. 1: Exemplary result sheet of one PANO examination. The result sheet displaying the contrast thresholds for each target, their mean and median value, the mean threshold values for quadrants and for zones, as well as the number of tests, the errors and the examination time
The average of mean threshold was $17.9 \pm 18.2$ (median $=8.48$; range: $2.19-63.2$ ) for PANO and $5.0 \pm 4.6$ (median $=2.74$; range: 1-15.8) for FDT. The overall mean of the total deviation was -10.8 \pm 10.2 (median $=-6.0$; range: -33.10 to 13.10 ) for HFA. The mean fundus area CDR was $0.44 \pm 0.23$ (median $=0.38$; range: $0.06-0.95$ ). The mean threshold value of PANO showed significant parametric and non-parametric correlations with mean threshold values of HFA-II, FDT-stimulus, and the fundus area-CDR. These are shown in Table 1 and in Figure 2.

\section{Glaucoma Discrimination}

Considering the above cut-off values, HFA detected 160 eyes with glaucoma, 53 eyes with borderline glaucoma, and 6 normal eyes, whereas PANO detected 144 eyes with glaucoma, 55 eyes with borderline glaucoma, and 20 normal eyes. One hundred and twenty-five eyes were glaucoma and 3 eyes were normal with both devices, resulting in a sensitivity of $95 \%$ and a specificity of $60 \%$ after the elimination of borderline cases (Fig. 3 and Table 2).

\section{Spatial Correlation}

Figure 4 shows the correlation coefficient between the mean thresholds of PANO and the total deviation of HFA for all 54 targets separately. Except for the blind spot, which the HFA does not measure, the thresholds at all targets correlate strongly and significantly ( $p<0.001$ for all correlations).

\section{Effect of Patient Age}

Patient age showed practically no effect on the results of the total deviation for the HFA. On the other hand, a negative correlation with age was demonstrated for the mean thresholds of PANO and FDT-stimulus. Figure 5 shows the distribution of examination results according to patient age.

\section{Examination Time}

The mean examination time was $7.1 \pm 1.8$ minutes (range: $3.2-11.5$ ) for PANO, $4.7 \pm 1.3$ minutes (range: 1.9-7.9) for the FDT-stimulus, and $5.9 \pm 1.3$ minutes (range: $3.2-10.3$ ) for the HFA. The difference in mean examination time between PANO and HFA, and between PANO and FDT was highly significant $(p<0.001$ using the Wilcoxon

Table 1: Pearson correlation between PANO, FDT-stimulus, HFA-II, and the area CDR. The mean total deviation of the HFA-II is a negative value

\begin{tabular}{|c|c|c|c|c|c|c|}
\hline \multicolumn{7}{|c|}{ Correlation coefficient of mean values } \\
\hline & \multicolumn{3}{|c|}{ Parametric correlation } & \multicolumn{3}{|c|}{ Non-parametric correlation } \\
\hline & FDT & HFA & Area-CDR & FDT & HFA & Area-CDR \\
\hline PANO & 0.94 & -0.85 & 0.62 & 0.85 & -0.68 & 0.51 \\
\hline FDT & & -0.86 & 0.64 & & -0.70 & 0.53 \\
\hline HFA & & & -0.65 & & & -0.51 \\
\hline
\end{tabular}
explaining the negative correlation

\begin{tabular}{|c|c|c|c|c|c|c|}
\hline & \multicolumn{3}{|c|}{ Parametric correlation } & \multicolumn{3}{|c|}{ Non-parametric correlation } \\
\hline & FDT & HFA & Area-CDR & FDT & HFA & Area-CDR \\
\hline PANO & 0.93 & -0.84 & 0.61 & 0.80 & -0.68 & 0.49 \\
\hline FDT & & -0.84 & 0.61 & & -0.66 & 0.54 \\
\hline HFA & & & -0.65 & & & -0.51 \\
\hline
\end{tabular}

Correlation coefficient of median values

All correlations attained a significance of $p<0.001$. CDR, cup-to-disk ratio; FDT, frequency doubling technology; PANO, pattern-noise; HFA, Humphrey Field Analyzer 

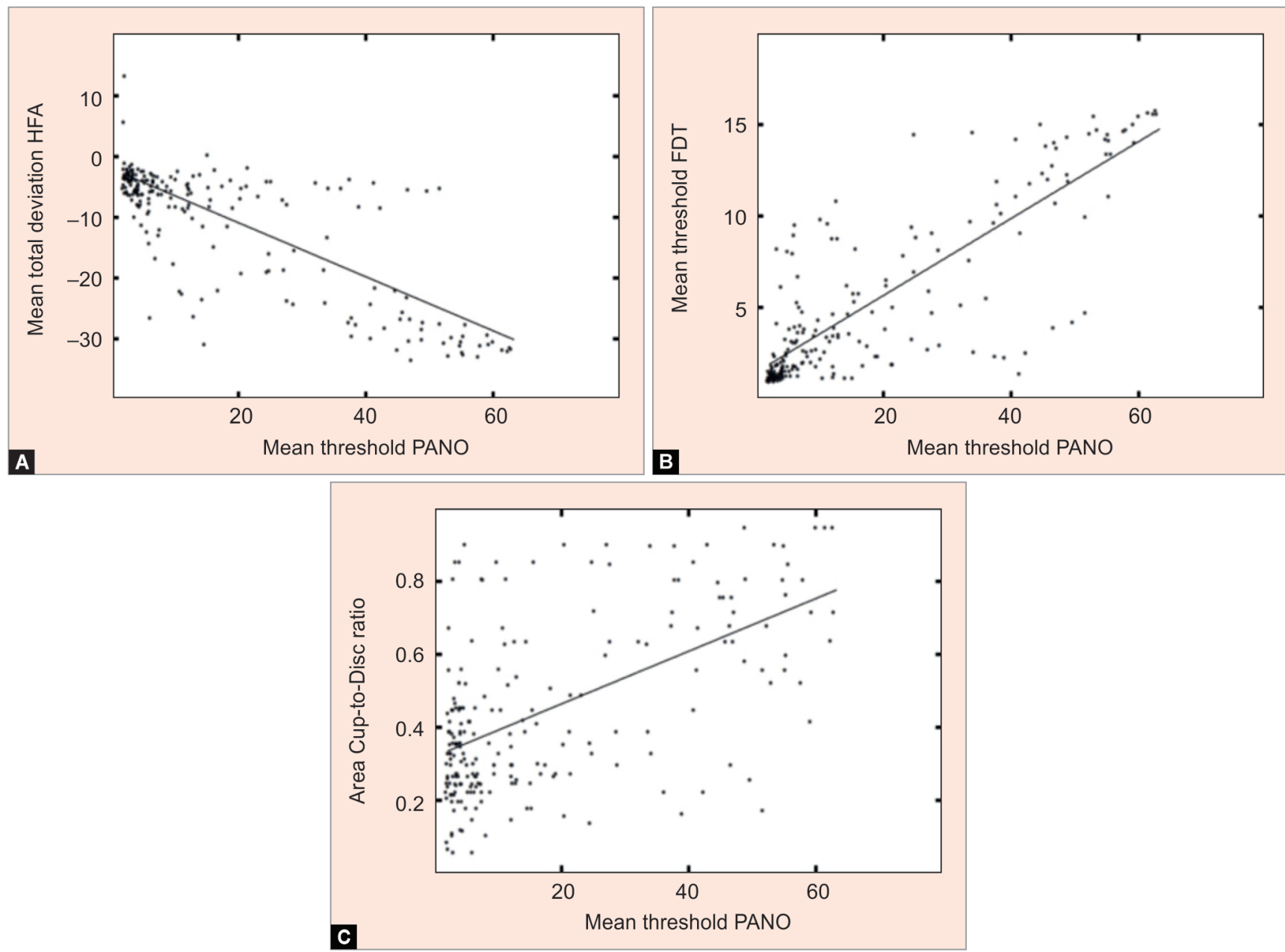

Figs 2A to C: Correlation of mean total deviation of the HFA, the FDT-stimulus, and the fundus area CDR with the mean threshold values of PANO. The correlation between the HFA and the PANO shows a negative slope, as the total deviation decreases while the visual field defect increases

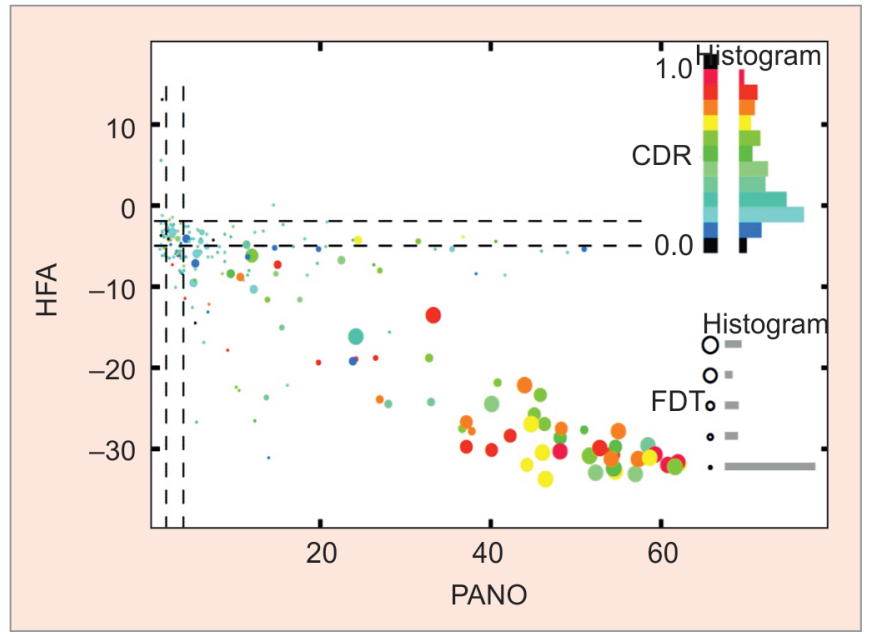

Fig. 3: Glaucoma discrimination criteria. The figure shows the combination of the four measured parameters (HFA, PANO, FDT-stimulus, and fundus area (DR) for glaucoma discrimination. For each patient the mean threshold for PANO is shown on the $x$-axis and the mean total deviation for HFA on the $y$-axis. The fundus area CDR is shown in colors as defined in the upper-right corner together with their histogram and the FDT values are coded in circle diameter as defined in the lower right corner together with their histogram. When we select cut-off values of $-3 \pm 1 \mathrm{~dB}$ for HFA (borderline: > $-2.0 \mathrm{~dB}$ and <-4.0 dB) and 4.0 \pm 1 for PANO (borderline: $>3$ and <5; see dashed lines), we obtain for HFA 160 glaucomas, 53 borderlines and 6 normals and for PANO 144 glaucomas, 55 borderlines and 20 normals. 125 eyes were glaucoma with both HFA and PANO and had a mean area CDR of 0.51 and an FDT value of 7.4. 3 eyes were normal with both devices and had a mean area CDR of 0.21 and an FDT value of 1.3. Note that because of a missing gold standard for glaucoma definition in general and because of missing normative data for the sub-Saharan African population, the preliminary discrimination between glaucoma, normal and borderline is based on literature. 
signed-rank test). The correlation between examination time for HFA, PANO, and FDT is shown in Figure 6.

\section{Comparison of Error Rate}

The mean percentage of error (false-positives) for the PANO examination was $4.95 \%$ and for FDT-stimulus $4.62 \%(p=0.025)$. The mean percentage of false-positives for HFA was $2.10 \%$. No comparison for error rates was calculated between PANO and HFA, as the criteria for false-positives are different.

Table 2: Glaucoma discrimination resulted in a sensitivity of $95 \%$ and a specificity of $60 \%$ for PANO if HFA was considered the gold standard and if borderline cases were eliminated

\begin{tabular}{lrlll}
\hline & & \multicolumn{3}{c}{ HFA } \\
\cline { 3 - 5 } & & Normal & Borderline & Glaucoma \\
\hline PANO & & 6 & 53 & 160 \\
Normal & 20 & 3 & 11 & 6 \\
Borderline & 55 & 1 & 25 & 29 \\
Glaucoma & 144 & 2 & 17 & 125 \\
\hline
\end{tabular}

Cut-off values were $-3 \pm 1 \mathrm{~dB}$ for HFA (borderline: $>-2.0 \mathrm{~dB}$ and $<-4.0$ $\mathrm{dB}$ ) and $4.0 \pm 1$ for PANO (borderline: $>3$ and $<5$ )

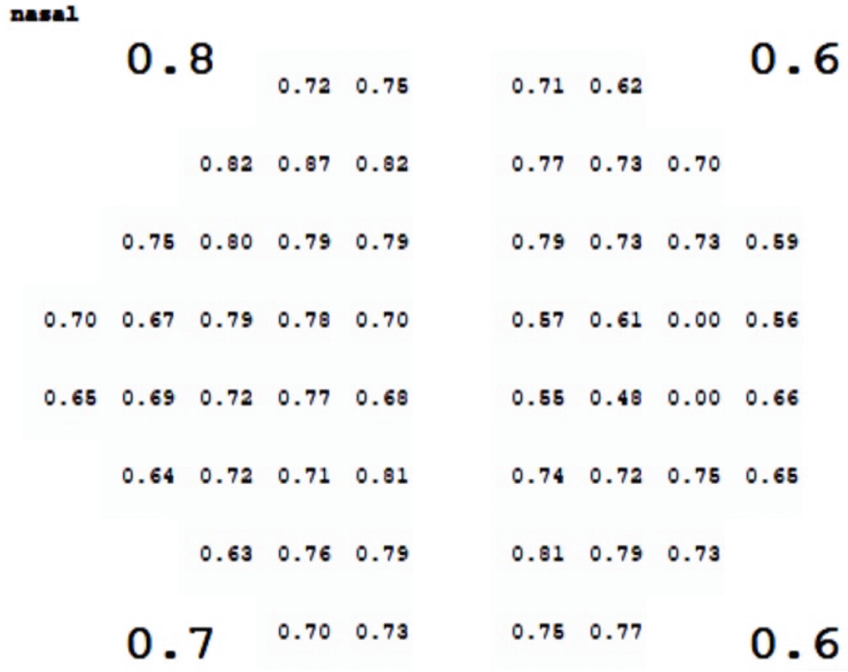

Fig. 4: Spatial distribution of correlation coefficients for all targets, and quadrants, separately. The correlation is highly significant at all points $(p<0.001)$, except at the blind spot, which is not measured in HFA. As the total deviation is a negative number, it was multiplied by -1
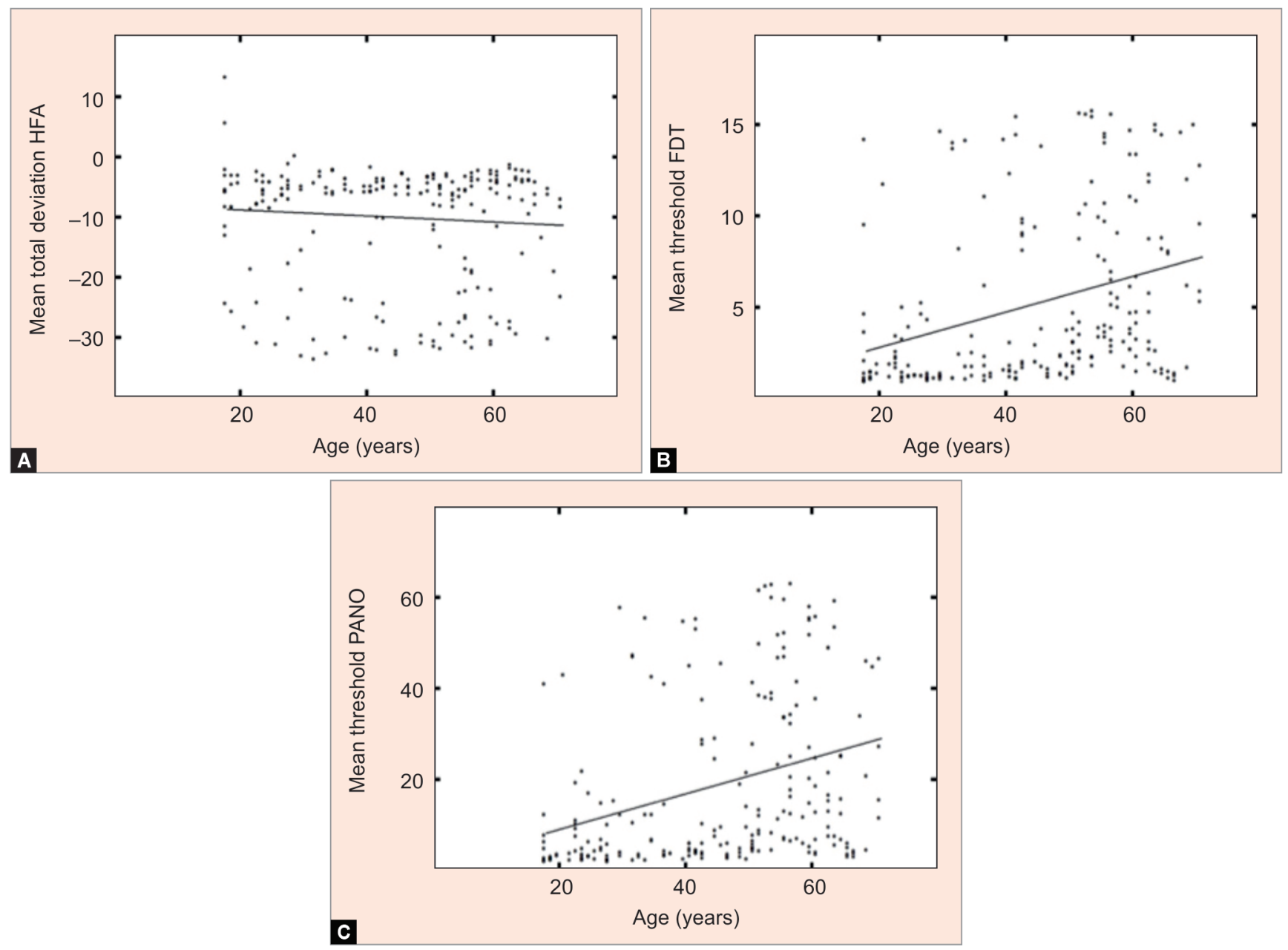

Figs 5A to C: Relation between mean total deviation of the HFA, mean threshold of PANO and mean threshold of FDT with patient age. The total deviation of the HFA shows the deviation from age-corrected, normal sensitivities and therefore shows no effect of age, whereas the mean threshold of the FDT-stimulus and PANO increases with age 

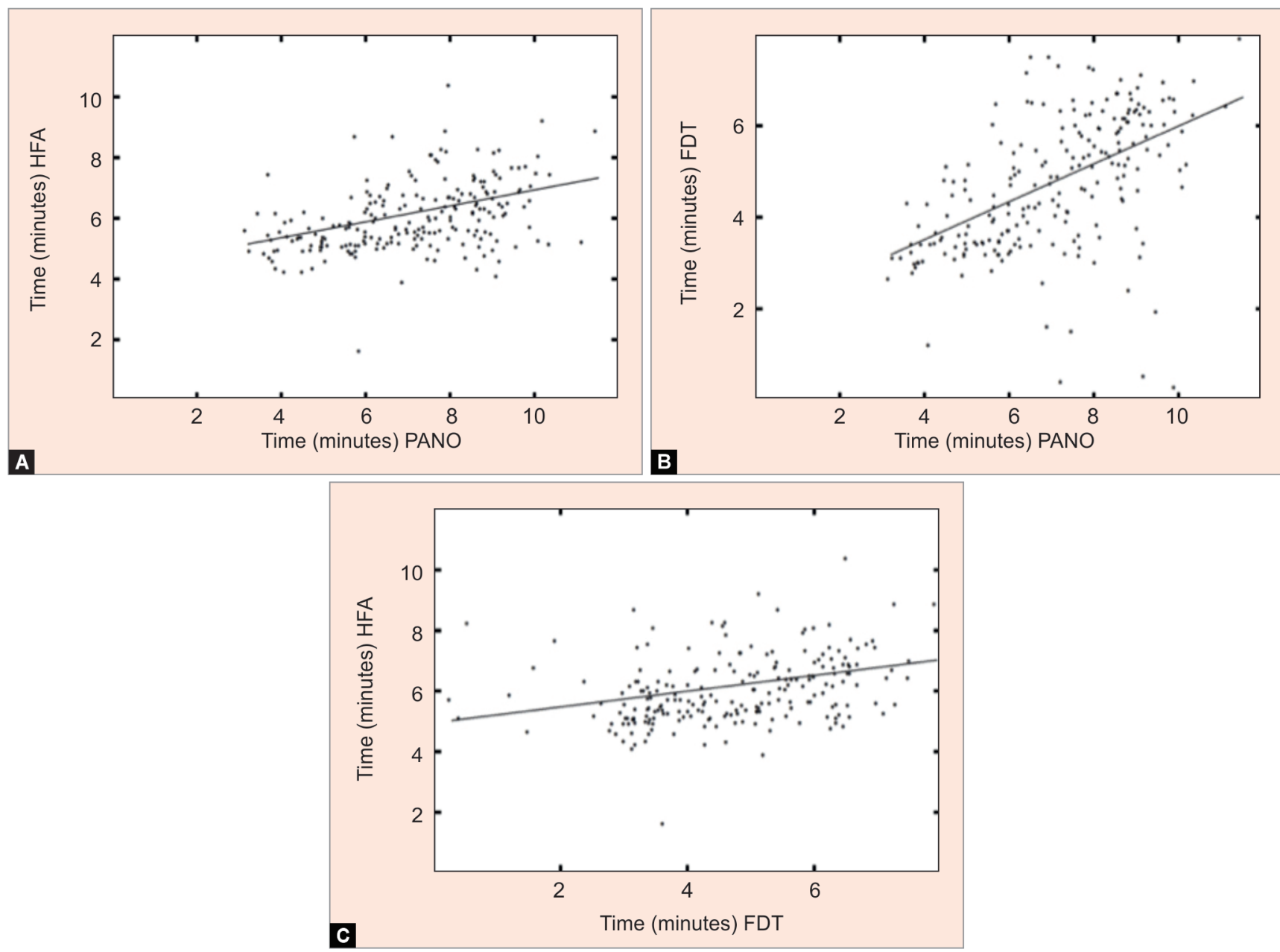

Figs 6A to C: Examination time. These figures show the correlation between the examination times for HFA with PANO, FDT with PANO and HFA with FDT

\section{Discussion}

Overall, the correlation of all four methods was highly significant. This means that PANO may successfully represent the same visual field defects as HFA-II. This claim is further underlined by the significant spatial correlation. Furthermore, PANO showed a reasonable examination time, averaging 1.2 minutes longer than the SITA standard on a 24-2 analyzer protocol. Also, there was a significant correlation between PANO and fundus area CDR, indicating the presence of glaucomatous optic nerve damage. On the other hand, the mean threshold of the FDT-stimulusessentially the same device as PANO with the same testing strategy but with a different stimulus-highly correlated with the mean threshold of PANO.

The sensitivity and specificity for PANO if HFA is considered the gold standard were $95 \%$ and $60 \%$, respectively. However, major limitations for these values are the lack of normative data in our population and the elimination of borderline cases. The cut-off values are preliminary and are based on data from the literature. Further studies are needed to verify these cut-off values in our population. Additionally, examinations with high error rates were not excluded in our study, which would have probably improved the sensitivity and specificity.
A close look at the graphic representation of mean thresholds in Figure 2 reveals a large number of eyes with a total deviation of less than -7 on HFA-II (134 eyes) and a mean threshold of less than 10 on PANO (113 eyes). It can be assumed that these eyes either have normal visual fields or very early glaucomatous defects. This finding with either method represents a tendency for a high falsepositive diagnosis of glaucoma and even possible over-treatment and may be linked to a lack of SAP in the above setting. The relatively large optic disks and disk cuppings observed in patients of African origin, ${ }^{15,16}$ as well as the systematic bilateral inclusion of eyes, may have contributed to this effect.

The decreased examination time of the FDT-stimulus in comparison to PANO was due to a lower number of possible contrasts (16) compared with PANO (63). To this end, fewer tests are needed for the FDT-stimulus to attain the contrast threshold.

With increased patient age, the mean thresholds of PANO and FDT also increased, whereas the mean threshold of HFA-II did not change. This is explained by the fact that HFA-II is age-corrected. For PANO and FDT, no such age correction was applied.

The statistical analysis was performed without taking into consideration that some patients had only one eye tested (25 patients) and the others (97 patients) had two eyes tested, causing a selection bias. However, this bias is marginal because statistics only 
considered the correlation between different glaucoma detection methods. A further limitation of the study is that the false-positive rate and the false-negative rate of PANO cannot be determined, due to the inability to definitely diagnose glaucoma in this setting and without follow-up. Another consideration is that only one clinician examined the fundus and that the estimation of the CDR is highly subjective, leaving room for a possible bias.

\section{Conclusion}

This study demonstrated that the computer-based glaucoma test PANO can display visual field defects as in HFA-II, FDT stimulus, and in correlation with the fundus area CDR. The results showed that PANO is a reliable technique to be used as a preliminary glaucoma test, particularly in low-income countries, which do not have enough glaucoma test machines. Further studies are needed to determine the sensitivity of PANO for early defects and for glaucoma progression.

\section{Clinical Significance}

Affordable and robust visual field devices are lacking in large parts of the developing world. Comparing them to established methods is a prerequisite to their clinical use.

\section{References}

1. Cook C, Foster P. Epidemiology of glaucoma: what's new? Canad J Ophthalmol 2012;47(3):223-226. DOI: 10.1016/j.jcjo.2012.02.003.

2. Quigley HA. Number of people with glaucoma worldwide. Br J Ophthalmol 1996;80(5):389-393. DOI: 10.1136/bjo.80.5.389.

3. Resnikoff S, Pascolini D, Etya'ale D, et al. Global data on visual impairment in the year 2002. Bullet World Health Organizat 2004;82(11):844-851. DOI: /S0042-96862004001100009.

4. Preußner P-R, Großmann A, Ngounou F, et al. Glaucoma screening in western Cameroon. Graefe's Archi Clin Experim Ophthalmol 2009;247(12):1671-1675. DOI: 10.1007/s00417-009-1166-7.
5. Buhrmann RR, Quigley HA, Barron Y, et al. Prevalence of glaucoma in a rural East African population. Investig Ophthalmol Visual Sci 2000;41(1):40-48.

6. Ntim-Amponsah CT, Amoaku WMK, Ofosu-Amaah S, et al. Prevalence of glaucoma in an African population. Eye 2004;18(5):491-497. DOI: 10.1038/sj.eye.6700674.

7. Öhnell H, Heijl A, Brenner L, et al. Structural and functional progression in the early manifest glaucoma trial. Ophthalmology 2016;123(6):1173-1180. DOI: 10.1016/j.ophtha.2016.01.039.

8. Olsen AS, Alberti M, Serup L, et al. Glaucoma detection with damato multifixation campimetry online. Eye 2016;30(5):731-739. DOI: 10.1038/eye.2016.25.

9. Ianchulev T, Pham P, Makarov V, et al. Peristat: a computer-based perimetry self-testforcost-effective population screening of glaucoma. Curr Eye Res 2005;30(1):1-6. DOI: 10.1080/02713680490522399.

10. Johnson CA, Thapa S, Kong YXG, et al. Performance of an iPad application to detect moderate and advanced visual field loss in Nepal. Am J Ophthalmol 2017;182:147-154. DOI: 10.1016/j. ajo.2017.08.007.

11. el-Khoury S, Hannen T, Dragnea DC, et al. Pattern noise (PANO): a new automated functional glaucoma test. Int Ophthalmol 2018;38(5):1993-2003. DOI: 10.1007/s10792-017-0690-4.

12. Arora KS, Boland MV, Friedman DS, et al. The relationship between better-eye and integrated visual field mean deviation and visual disability. Ophthalmology 2013;120(12):2476-2484. DOI: 10.1016/j. ophtha.2013.07.020.

13. Alencar LM, Medeiros FA. The role of standard automated perimetry and newer functional methods for glaucoma diagnosis and follow-up. Indian J Ophthalmol 2011;59(Suppl 1):S53-S58. DOI: 10.4103/03014738.73694.

14. Quigley HA. Identification of glaucoma-related visual field abnormality with the screening protocol of frequency doubling technology. Am J Ophthalmol 1998;125(6):819-829. DOI: 10.1016/ s0002-9394(98)00046-4.

15. Tsai CS, Zangwill L, Gonzalez C, et al. Ethnic differences in optic nerve head topography. J Glauc 1995;4(4):248-257. DOI: 10.1097/00061198 199508000-00006.

16. Analyzer OD. Racial differences in optic nerve head parameters. Arch Ophthalmol 1989;107(6):836-839. DOI: 10.1001/archo pht.1989.01070010858029. 